\title{
POCUS predicts prognosis in cardiac arrest
}

\section{Elizabeth Lalande (1), MD*; Michael Y. Woo, MD ${ }^{\dagger}$}

Point-of-care ultrasound (POCUS) is a core application of emergency medicine training and is now internationally recognized as a diagnostic tool in resuscitation. ${ }^{1-2}$ The 2015 update of the American Heart Association (AHA) Guidelines for Cardiopulmonary Resuscitation (CPR) and Emergency Cardiovascular Care (ECC) states that POCUS may be used during CPR of cardiac arrest patients, although its usefulness has not been well established. ${ }^{3}$ Beckett et al. seeks to define more precisely how best POCUS can contribute to the prognosis assessment of patients in cardiopulmonary arrest. The authors evaluated the prognostic value of POCUS in comparison with electrocardiogram (ECG) but also by combining it with the latter when treating patients in cardiopulmonary arrest.

We know that traumatic cardiac arrest differs from atraumatic cardiac arrest in terms of prognosis and etiologies. $^{4-5}$ Furthermore, we also know that a much favorable prognosis is associated with shockable rhythms in cardiac arrest compared with non-shockable rhythms. ${ }^{6}$ The fact that the authors precisely defined their population by choosing atraumatic cardiac arrest with non-shockable rhythms allows clinicians to use these results to this specific patient population. Despite the methodological limitations of a health-record review of only 180 cases, the authors are able to add to the body of knowledge regarding prognostication in cardiac arrest. Both ECG and POCUS performed better to predict negative outcomes, death or failure to achieve return of spontaneous circulation (ROSC), survival to hospital admission and survival to hospital discharge, compared with positive ones. For ROSC outcome, when using POCUS for patients in cardiac arrest, if there was absence of cardiac activity, there was a $96.2 \%$ chance that the resuscitation would be unsuccessful and result in death. When using ECG alone, in patients in asystole, they found a $82.7 \%$ chance that the resuscitation would be unsuccessful. When compared, POCUS performed better than ECG as a predictor of death for all three outcomes. However, the more eloquent results were found when combining ECG and POCUS (Figure 1). Authors found a sensitivity of $98.2 \%$ for patients who had asystole on ECG and no cardiac activity on POCUS, demonstrating the strength of this combination for this precise subgroup.

Atkinson et al. ${ }^{7}$ recently published a study in which authors state that patients with cardiac activity on POCUS had improved clinical outcomes as compared with patients not receiving POCUS and patients with no activity on POCUS. These patients with cardiac activity received longer resuscitation with higher rates of intervention as compared with those without cardiac activity on POCUS or when it was not performed. It will be a challenge to evaluate the favorable outcomes of pulseless electrical activity patients with cardiac activity on POCUS while minimizing the impact of POCUS itself on the results.

POCUS could aid in predicting death among patients with asystole and without cardiac activity while predicting favorable clinical outcomes in the subgroup of patients with pulseless electrical activity and cardiac activity on POCUS. ${ }^{8}$ The importance of ongoing research cannot be understated because POCUS is potentially not without harm. The 2015 update of the AHA guidelines for CPR and ECC guidelines states specifically that POCUS should not interfere with the standard cardiac arrest treatment protocol. ${ }^{3}$ A previous study demonstrated delays in CPR when POCUS was used during pulse checks. ${ }^{9}$ Subsequently, another study demonstrated the importance and effective use of a

From the *Department of Emergency Medicine, Université Laval, Centre Hospitalier de I'Université Laval (CHUL), Quebec, QC; and the †Department of Emergency Medicine, University of Ottawa, Ottawa Hospital Research Institute, Ottawa, ON.

Correspondence to: Dr. Elizbeth Lalande, Urgence du CHUL, CHU de Québec, secrétariat du Service d'Urgence, 2705 Boulevard Laurier, Québec, Québec, G1V 4G2; Email: Ialande.elizabeth@gmail.com

(c) Canadian Association of Emergency Physicians

CJEM 2019;21(6):689-690

DOI 10.1017/cem.2019.436 


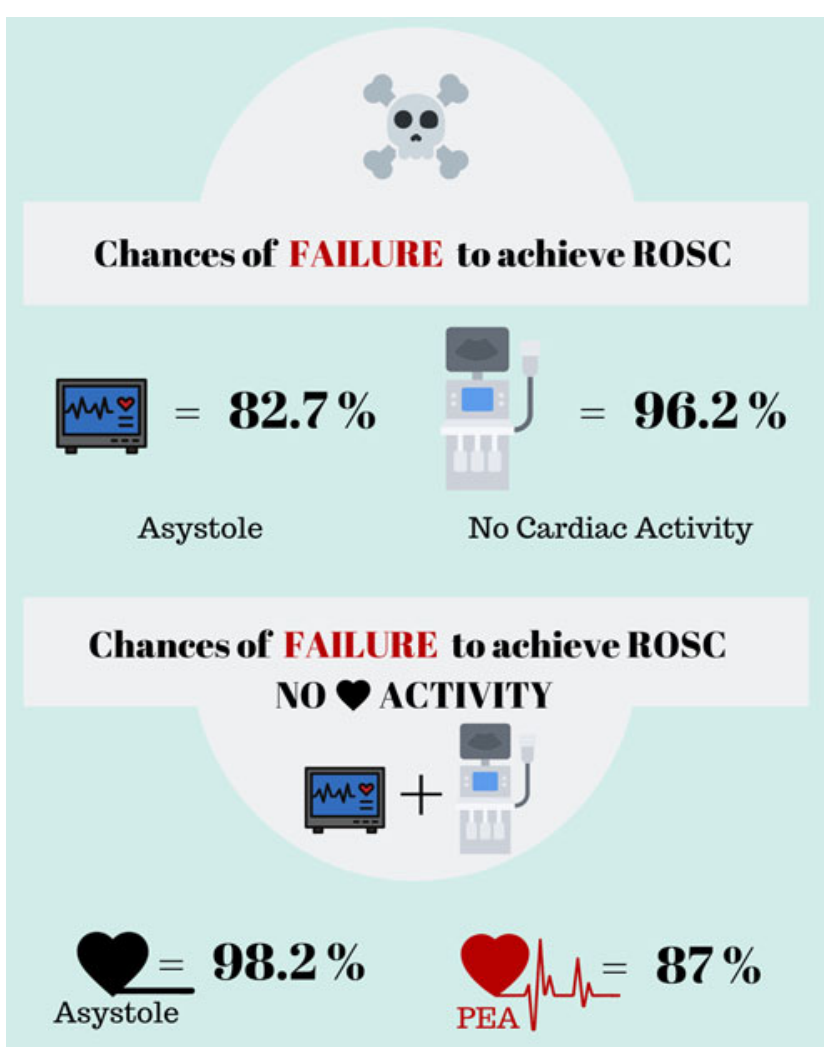

Figure 1. Sensitivity of initial electrocardiogram rhythm (ECG), initial point-of-care ultrasound (PoCUS) findings overall, and PoCUS findings in patients with ECG asystole, and pulseless electrical activity, for the predication of no return of spontaneous circulation.

POCUS-integrated cardiac arrest protocol to address delays in CPR with POCUS. ${ }^{10}$

Cardiac arrest care continues to evolve and with that the role of POCUS. Surely, with the evidence that exists now, POCUS has a role in predicting death when there is no cardiac activity. However, patients with cardiac arrest are complex, and there is no single best test to determine their prognosis. There still remain many questions to be answered. How should we define cardiac activity on POCUS? When should we perform POCUS when trying to evaluate the prognosis of our patient? Does it matter? What is the best combination of tests we should use to determine a patient prognosis while in cardiac arrest? Beckett's results help us take one step further into answering these important clinical questions. POCUS is essential to the evolution of cardiac arrest care.
Keywords: Ultrasound, cardiac arrest, emergency medicine

Competing interests: None declared.

\section{REFERENCES}

1. The Emergency Medicine Milestone Project. Accreditation Council for Graduate Medical Education; 2012. Available at: https://www.acgme.org/Portals/0/PDFs/Milestone/EmergencyMedicineMilestones.pdf (accessed March 1, 2018).

2. Atkinson P, Bowra J, Milne J, et al. International Federation for Emergency Medicine Consensus Statement: sonography in hypotension and cardiac arrest (SHoC): an international consensus on the use of point of care ultrasound for undifferentiated hypotension and during cardiac arrest. CFEM 2017;19:459-70.

3. Link MS, Berkow LC, Kudenchuk PJ, et al. Part 7: adult advanced cardiovascular life support: 2015 American Heart Association Guidelines Update for Cardiopulmonary Resuscitation and Emergency Cardiovascular Care. Circulation 2015;132:S444-64.

4. Soar J, Perkins GD, Abbas G, et al. European Resuscitation Council Guidelines for Resuscitation 2010 Section 8. Cardiac arrest in special circumstances: electrolyte abnormalities, poisoning, drowning, accidental hypothermia, hyperthermia, asthma, anaphylaxis, cardiac surgery, trauma, pregnancy, electrocution. Resuscitation 2010;81(10):1400-33.

5. Hopson LR, Hirsh E, Delgado J, et al. Guidelines for withholding or termination of resuscitation in prehospital traumatic cardiopulmonary arrest: joint position statement of the National Associations of EMS Physicians and the American College of Surgeons Committee on trauma. $7 \mathrm{Am}$ Coll Surg 2003;196(1):106-12.

6. Chan PS, McNally B, Tang F, Kellerman A. CARES Surveillance Group. Recent trends in survival from out-of-hospital cardiac arrest in the United States. Circulation 2014;130:1876-82.

7. Atkinson P, Beckett N, French J, et al. Does point-of-care ultrasound use impact resuscitation length, rates of intervention, and clinical outcomes during cardiac arrest? A study from the sonography in hypotension and cardiac arrest in the Emergency Department (SHoC-ED) Investigators. Cureus 2019;11(4):e4456.

8. Lalande E, Burwash-Brennan T, Burns $\mathrm{K}$, et al. Is point-of-care ultrasound a reliable predictor of outcome during atraumatic, non-shockable cardiac arrest? A systematic review and meta-analysis from the SHoC Investigators. Resuscitation 2019;139:159-66.

9. Huis in't Veld MA, Allison MG, Bostick DS, et al. Ultrasound use during cardiopulmonary resuscitation is associated with delays in chest compressions. Resuscitation 2017;11:95-8.

10. Clattenburg EJ, Wroe PC, Gardner K, et al. Implementation of the cardiac arrest sonographic assessment (CASA) protocol for patients with cardiac arrest is associated with shorter CPR pulse checks. Resuscitation 2018;131:69-73. 